\title{
Identification of Time-Varying Cortico-cortical and Cortico- Muscular Coherence during Motor Tasks with Multivariate Autoregressive Models
}

\author{
Alba Xifra-Porxas, Kyriaki Kostoglou, Sara Larivière, Guiomar Niso, Michalis Kassinopoulos, Marie- \\ Hélène Boudrias and Georgios D. Mitsis, Senior Member, IEEE
}

\begin{abstract}
Neural populations coordinate at fast subsecond time-scales during rest and task execution. As a result, functional brain connectivity assessed with different neuroimaging modalities (EEG, MEG, fMRI) may also change over different time scales. In addition to the more commonly used sliding window techniques, the General Linear Kalman Filter (GLFK) approach has been proposed to estimate time-varying brain connectivity. In the present work, we propose a modification of the GLFK approach to model timevarying connectivity. We also propose a systematic method to select the hyper-parameters of the model. We evaluate the performance of the method using MEG and EMG data collected from 12 young subjects performing two motor tasks (unimanual and bimanual hand grips), by quantifying time-varying cortico-cortical and corticomuscular coherence (CCC and $\mathrm{CMC}$ ). The $\mathrm{CMC}$ results revealed patterns in accordance with earlier findings, as well as an improvement in both time and frequency resolution compared to sliding window approaches. These results suggest that the proposed methodology is able to unveil accurate time-varying connectivity patterns with an excellent time resolution.
\end{abstract}

\section{INTRODUCTION}

Stationary autoregressive (AR) models have been used extensively in the past to model neuroscience data [1]. However, measured brain activity and couplings change rapidly with time [2], for example due to event-related perturbations, learning and feedback. This implies that timevarying system formulations are more appropriate to model these non-stationarities. Several studies have successfully applied time-varying AR models in different neuroscience applications, for instance in task-based EEG/MEG [3]-[6].

A. Xifra-Porxas and M. Kassinopoulos are with the Graduate Program in Biological and Biomedical Engineering, McGill University, Montréal, Canada.

K. Kostoglou is with the Department of Electrical and Computer Engineering, McGill University, Montréal, Canada.

S. Larivière is with the Integrated Program in Neuroscience, McGill University, Montréal, Canada.

Guiomar Niso is with the McConnell Brain Imaging Center, Montreal Neurological Institute, Montréal, Canada.

M-H. Boudrias is with the Department of Physical and Occupational Therapy, McGill University, Montréal, Canada.

A. Xifra-Porxas and M-H. Boudrias are with the Center for Interdisciplinary Research in Rehabilitation of Greater Montreal (CRIR), Montréal, Canada.

G. D. Mitsis is with the Department of Bioengineering, McGill University, Montréal, Canada (corresponding author, phone: +1 514.398.4344, email: georgios.mitsis@mcgill.ca).

This work was supported by the the Fonds Recherche Nature et Technologies Quebec (Team Grant awarded to GDM and MHB) and the Natural Sciences and Engineering Research Council of Canada (Discovery Grant awarded to GDM).
The preferred method is the General Linear Kalman Filter (GLFK) [6] because it allows the estimation of highdimensional multiple-trial time-varying multivariate autoregressive models (TV-MVAR).

Synchronized oscillations between the motor cortex and the motor units of the contralateral muscles have been extensively investigated since the early 90 s, and have been interpreted as a sign of neural coordination. A commonly used approach to study the synchrony between signals is through coherence estimation. Motor cortex oscillations are coherent with activity in the contralateral muscles during sustained contraction around $20 \mathrm{~Hz}$ [7]. This synchronization is usually referred as cortico-muscular coherence (CMC). Classical methods to estimate CMC are through Fast Fourier Transform or bivariate MVAR models, used in a slidingwindow fashion in cases where time-varying patterns are investigated. The most significant caveat of these approaches is that they simply allow a pairwise analysis, thus only the coupling between two signals can be analyzed simultaneously. However, a multivariate approach that simultaneously estimates coherence between brain areas as well as coherence between neural and muscle activity may be more suitable, since premotor areas have also been reported to show CMC [8][9]. Therefore, in the present paper we propose a modification of the multivariate GLFK method and we evaluate its performance using MEG and EMG recordings. This methodology accounts for fast variations in the AR estimates by introducing multiple update coefficients in the methodology. Also, we propose a systematic approach to choose the hyper-parameters of the model.

\section{METHODS}

\section{A. Subjects}

Twelve healthy right-handed student volunteers $(24.2 \pm$ 2.8 years) participated in the study. The study was approved by the McGill University Ethical Advisory Committee and All subjects signed a written informed consent in accordance with the Declaration of Helsinki.

\section{B. Experimental paradigm}

Prior to MEG scanning, six surface electromyography (sEMG) electrodes were placed on each subject (first dorsal interosseous left/right, extensor digitorum communis left/right, flexor carpi radialis left/right), and the maximum voluntary contraction (MVC) for each participant was recorded. The task carried out inside the MEG scanner consisted of three periods of 5-min resting-state, alternated with two motor tasks (Fig. 1). The first motor task was a 


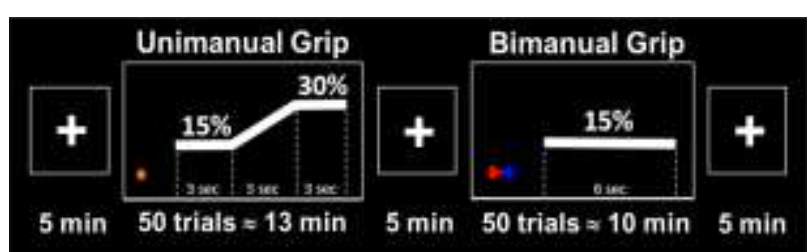

Figure 1. Illustration of the experimental protocol.

unimanual isometric right-hand grip in which the subjects had to apply force to track a ramp target as accurately as possible. The target force was set at $30 \%$ of their MVC. A single trial lasted 11 seconds, and it was repeated 50 times ( 13 minutes). The second motor task was a bimanual steady isometric hand grip at $15 \%$ of their MVC. A single trial lasted 8 seconds, during which the grip was sustained for 6 seconds, and it was repeated 50 times ( $\sim 8$ minutes). For both tasks, the inter-trial interval jittered between 3-5 seconds and a fixation cross was shown on the screen. Visual feedback was provided.

\section{MEG data acquisition and pre-processing}

MEG recordings were acquired with a CTF 275 system located at the Montreal Neurological Institute, with the subjects in a seated position. The 3D digitization of the head shape was done using a Polhemus Fastrak device, using approximately 100 head points that were uniformly distributed. An anatomical brain image was obtained for each subject on a 1.5T MRI. All signals were amplified and digitized at a sampling rate of $2.4 \mathrm{kHz}$. Offline, the signals were processed with Brainstorm [10]. MEG data were filtered using a notch filter at 60, 120 and $180 \mathrm{~Hz}$, bandpassed from 1 to $150 \mathrm{~Hz}$, and down-sampled to $120 \mathrm{~Hz}$. Artifacts due to heartbeats and blinks were removed using signal space projection. All single MEG trials were inspected visually and trials with artifacts were removed from further analysis. This procedure left $\geq 35$ trials per subject for each motor task. EMG data were high-passed at $30 \mathrm{~Hz}$ and fullwave rectified. The pre-processed MEG data were projected onto a grid spanning the entire brain using a linearlyconstrained minimum variance beamformer [11]. Data from the unimanual task were epoched from -2 to +13 seconds, and data from the bimanual task were epoched from -2 to +10 seconds. Time 0 indicates onset of the visual cue for analysis. Thus, each epoch consisted of 1800 samples for the unimanual task, and 1440 samples for the bimanual task.

We examined 9 regions of interest (ROIs) extracted from MEG source time-courses from brain areas thought to be involved in motor control. These areas are the primary motor hand representational area (M1, left and right), the dorsal premotor cortex (PMd, left and right), the ventral premotor cortex (PMv, left and right), the superior parietal cortex (SP, left and right), and the supplementary motor area (SMA). A well-known problem for MEG coherence estimation is the spurious correlations between the inferred cortical sources. Several methods have been proposed to correct this issue. One such method is to orthogonalize the time-series prior to connectivity estimation (signal leakage correction) [12][13]. However, such methods can be overconservative, since zerolag as well as lagged correlations are removed. Hence, we performed coherence estimation considering two scenarios: correcting ROI time-courses for signal leakage effects using a multivariate approach [13], and not correcting for these effects. All signals were normalized prior to further analysis.

\section{MEG source data analysis}

In discrete time, a TV-MVAR of order $p$ is defined as:

$$
\mathbf{Y}_{t}=\sum_{i=1}^{p} \mathbf{A}_{t-i} \mathbf{Y}_{t-i}+\mathbf{E}_{t}
$$

where $\mathbf{Y}_{t} \in \mathbb{R}^{M \times K}=\left[\begin{array}{lll}\mathbf{y}_{t}^{1} & \ldots & \mathbf{y}_{t}^{K}\end{array}\right]$ is a matrix of $M$ signals of interest for $K$ trials at time $t ; \mathbf{A}_{t-i} \in \mathbb{R}^{M \times M}$ is a time-dependent matrix of autoregressive coefficients for lag $i$; and $\mathbf{E}_{t} \in \mathbb{R}^{M \times K}=\left[\begin{array}{lll}\boldsymbol{\varepsilon}_{t}^{1} & \ldots & \boldsymbol{\varepsilon}_{t}^{K}\end{array}\right]$ is assumed to be a matrix of uncorrelated zero-mean white noise processes.

The $M$ signals are represented as a linear combination of past values of all signals, including their own. The autoregressive coefficients of the model vary with time, and represent the influence of one signal on another. The model coefficients are estimated using the GLFK technique [6]. Specifically, one TV-MVAR model was fitted to each subject using all trials, which assumes all trials come from the same stochastic ensemble. The measurement noise was assumed to be instantaneously uncorrelated across signals, as in [14], which offers significant computational advantages. To improve the Kalman filter performance, the innovation covariance matrix was fixed to a constant value, whereas the state covariance matrix was updated adaptively based on the following equation:

$$
\mathbf{Q}_{t}=(\mathbf{I}-\boldsymbol{\lambda}) \mathbf{Q}_{t-1}+\boldsymbol{\lambda}\left(\mathbf{I} \otimes \operatorname{diag}\left(\mathbf{E}_{t} \mathbf{E}_{t}^{\mathrm{T}}\right)\right)
$$

where $\mathbf{Q}_{t} \in \mathbb{R}^{M^{2} p \times M^{2} p}$ is the state covariance matrix, $\lambda \in \mathbb{R}^{M^{2} p \times M^{2} p}$ is a diagonal matrix with update coefficients that are assigned to each AR parameter, $\mathbf{I} \in \mathbb{R}^{M^{2} p \times M^{2} p}$ is the identity matrix, and $\otimes$ represents the Kronecker product. Using multiple update coefficients allows to accurately track possible different rate of variations for each parameter, as proposed in our previous work [15][16]. Note that the matrix $\operatorname{diag}\left(\mathbf{E}_{t} \mathbf{E}_{t}^{\mathrm{T}}\right)$ needs to be expanded to form an $M^{2} p \times M^{2} p$ block-diagonal matrix. Offline, the AR coefficients are smoothed using a Kalman smoother approach [3]. Finally, time-varying coherence is extracted by the estimated TVMVAR coefficients [17]. In this study, we considered 9 ROIs and the mean of the 3 rectified EMG signals from the active hand. Hence, the number of signals is equal to 10 and 11 for the unimanual and bimanual tasks, respectively. The extracted time-varying coherence describes changes in coupling between the ROIs and active muscles (TV-CMC) and between ROIs in the cortex (TV-CCC).

Model order selection is a crucial step that may significantly affect the coherence estimates [18]. We propose to use a mixed integer genetic algorithm (GA) [19] instead of arbitrarily selecting a model order. A GA is a heuristic technique based on a natural selection process that mimics evolutionary biology. It adaptively searches for a global minimum of an optimization problem. At each iteration, a candidate solution is tested against the objective function, which in our case is the Akaike Information Criterion (AIC). AIC provides a trade-off between the goodness of fit of a model and its complexity, since it includes a penalty function of the number of coefficients to avoid overfitting. The hyperparameters being searched are the model order $p$ and the 
update coefficients of the covariance matrices of the innovation process and the parameter estimates.

\section{RESULTS AND DISCUSSION}

\section{A. Time-varying Cortico-Muscular Coherence}

Previous studies have shown increased CMC in the beta frequency range $(15-35 \mathrm{~Hz})$ during a maintained contraction, followed by a reduction in beta CMC during the ramp period of a contraction [20]. In our experiment, we observed that TV-CMC in the beta band was not a consistent finding among our cohort. Further, there was inhomogeneity in amplitude and peak frequency among the cohort subjects, which made pooling of the data not feasible. This is not unexpected since high inter-subject variability has been previously reported [21]. In the unimanual task, TV-CMC was only observed in four subjects. Nonetheless, these subjects presented the anticipated TV-CMC task-modulation, as can be seen in Fig. 2a, b. Specifically, CMC emerged during hold periods, and decreased during the dynamic ramp phase. Interestingly, during the bimanual task, TV-CMC was observed at least for one hand in ten out of twelve subjects. CMC emerged during the maintained contraction, and appeared to increase toward the end of the trial, as shown in Fig. 2c, d. This may be explained because it was difficult to coordinate a sustained hold with both hands, which was generally accomplished in the last seconds of the trial. The cortical area that showed the highest values of TV-CMC was M1, but similar patterns were also observed for PMd and SMA. TV-CMC results did not change whether leakage correction was applied or not.

For the sake of comparison, we carried out a traditional sliding-window analysis. An MVAR model was estimated in each window, with $90 \%$ overlap between windows. Results are shown in Fig. 3 for model orders 2, 5 and 10, and window sizes of $0.5,1,1.5,2$ and 2.5 seconds. The TV-CMC pattern is visible in most subfigures, yet the trade-off between frequency and time resolution is also evident. Higher model order results in enhanced frequency resolution, and smaller window size results in enhanced time resolution. Hence, it is difficult to decide which combination reflects the true underlying dynamics, thus requiring an arbitrary choice. Contrarily, the proposed methodology uses the GA to choose the model order and the update coefficients, which optimize the rate of change of each AR coefficient and implicitly improves the time-frequency resolution.

\section{B. Time-varying Cortico-Cortical Coherence}

Regarding the analysis where signal leakage correction was not applied, high values of coherence across all frequencies were observed between multiple ROIs. This is not surprising since some of the ROIs are very close to each other (e.g. M1 and PMd). On the other hand, the analysis with leakage correction did not reveal any consistent patterns. This negative finding does not seem to be due to the methodology, since we were able to demonstrate previously shown patterns in TV-CMC driven by the motor task, and has also been reported in other studies [22].

\section{Model order}

The model order selected by the GA ranged between 2 and 8 for different subjects. To verify the absence of possible underfitting, we repeated the analysis varying the model order from 2 to 20 , with a step of 1 . The results for both TV-CMC and TV-CCC did not change significantly.

(a)

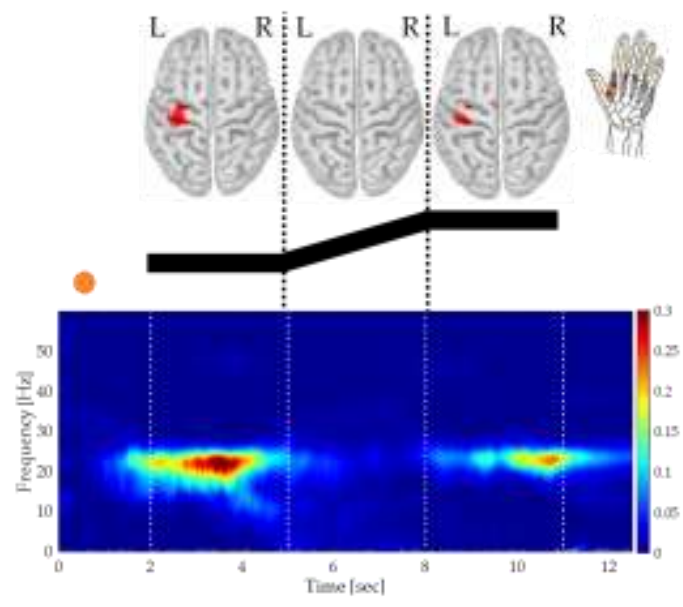

(c)

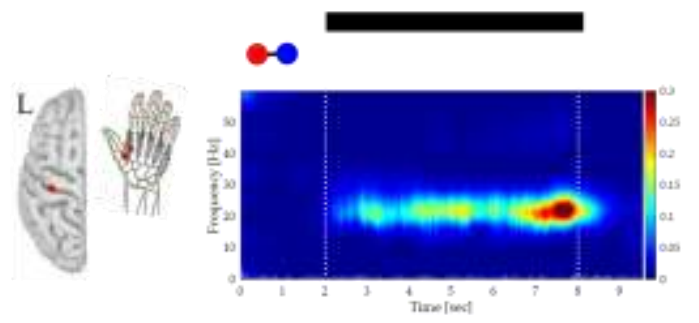

(d)

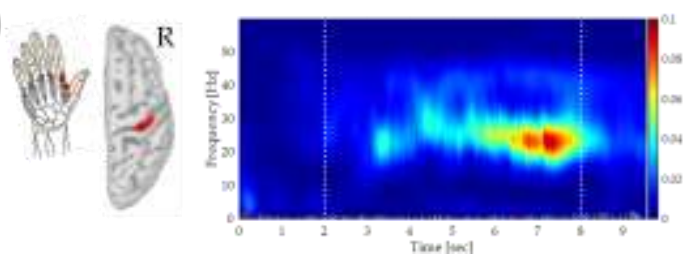

Figure 2. Results from a representative subject. Unimanual task: (a) Beta band (15-28 Hz) CMC averaged across task periods, (b) TV-CMC between right-hand EMGs and contralateral M1. Bimanual task: Beta band CMC averaged across task periods (left images), and TV-CMC between EMGs and contralateral M1 (right images) for (c) right hand, and (d) left hand.

\section{CONCLUSION}

We presented an accurate quantification method for timevarying connectivity dynamics, which combines MVAR models with Kalman-based estimation along with a genetic algorithm for model order selection. The proposed method achieves excellent time resolution, while removing the need of arbitrary model selection. The replication of beta-band TV-CMC modulation during the unimanual task validates that the methodology is able to unveil time-varying couplings. Further, the inclusion of all ROIs that may be related to the emergence of CMC in the estimation of TVCMC overcomes the problem of the "hidden-inputdilemma", because signals not included in the model can cause misleading interaction results. A potential limitation of CMC, possibly regardless of the methodology, is its variability usually observed across individuals [21]. This restricts its clinical application, as the absence of $\mathrm{CMC}$ does not necessarily imply pathology given that $\mathrm{CMC}$ is not always observed in healthy subjects. 


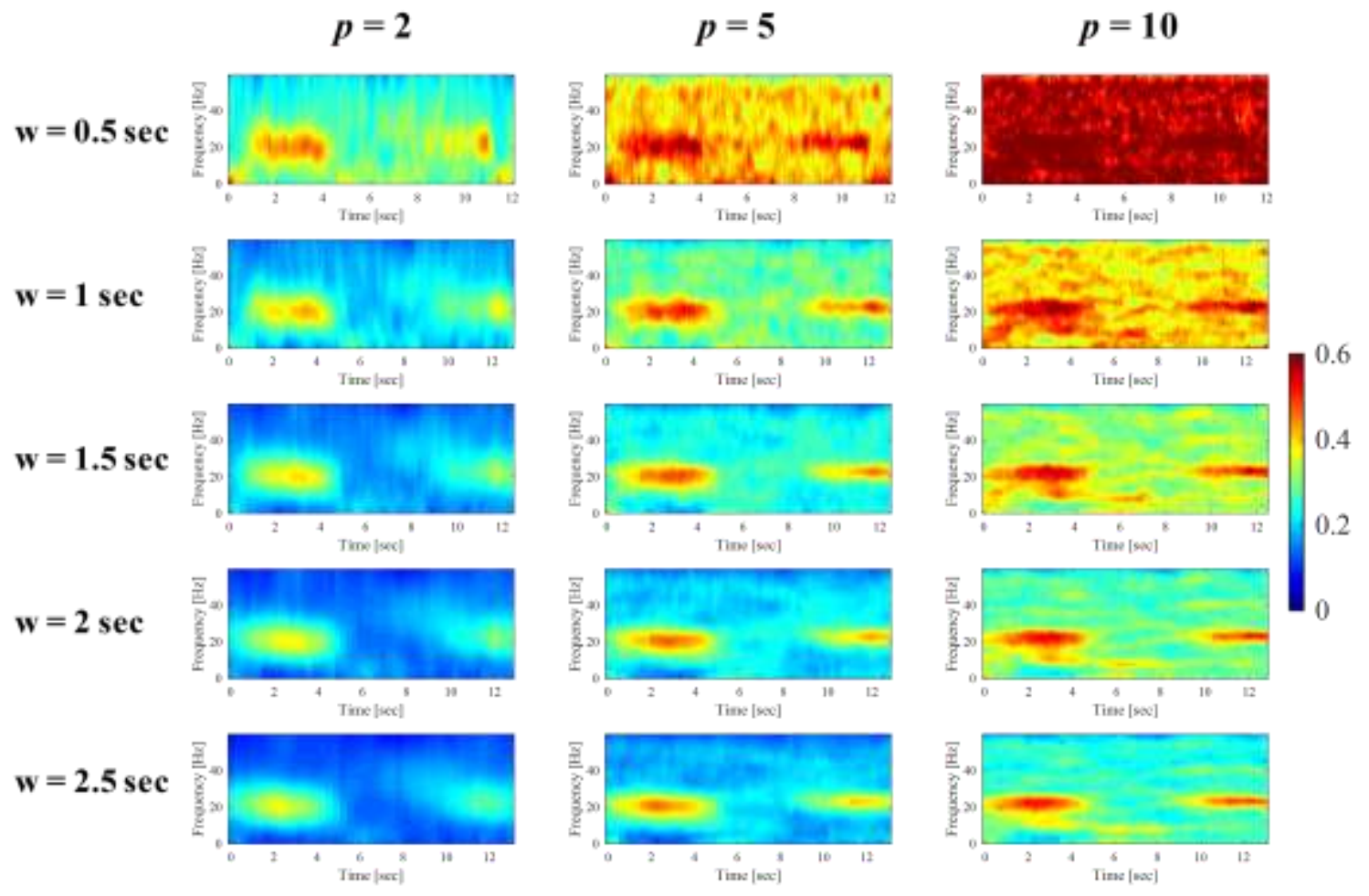

Figure 3. Sliding-window analyses with varying model order and window size. Data is from the same subject as in Fig. 2.

\section{REFERENCES}

T. Ozaki, Time series Modeling of Neuroscience Data. 2012. G. Buzsáki, Rhythms of the Brain. 2009.

M. P. Tarvainen, J. K. Hiltunen, P. O. Ranta-Aho, and P. A. Karjalainen, "Estimation of Nonstationary EEG with Kalman Smoother Approach: An Application to Event-Related Synchronization (ERS)," IEEE Trans. Biomed. Eng., vol. 51, no. 3, pp. 516-524, 2004.

[4] L. Astolfi, F. Cincotti, D. Mattia, F. De Vico Fallani, A. Tocci, A Colosimo, S. Salinari, M. G. Marciani, W. Hesse, H. Witte, M. Ursino, M. Zavaglia, and F. Babiloni, "Tracking the time-varying cortical connectivity patterns by adaptive multivariate estimators," IEEE Trans. Biomed. Eng., 2008.

B. Leung, P. Cheung, B. A. Riedner, G. Tononi, and B. D. Van Veen, "Estimation of Cortical Connectivity From EEG using State-Space Models," IEEE Trans. Biomed. Eng., vol. 57, no. September, pp. 2122-2134, 2010.

[6] T. Milde, L. Leistritz, L. Astolfi, W. H. R. Miltner, T. Weiss, F. Babiloni, and H. Witte, "A new Kalman filter approach for the estimation of high-dimensional time-variant multivariate AR models and its application in analysis of laser-evoked brain potentials," Neuroimage, vol. 50, no. 3, pp. 960-969, 2010

[7] S. N. Baker, "Oscillatory interactions between sensorimotor cortex and the periphery," Curr. Opin. Neurobiol., vol. 17, no. 6, pp. 649-655, 2007.

[8] J. Gross, B. Pollok, M. Dirks, L. Timmermann, M. Butz, and A. Schnitzler, "Task-dependent oscillations during unimanual and bimanual movements in the human primary motor cortex and SMA studied with magnetoencephalography," Neuroimage, vol. 26, no. 1, pp. 91-98, 2005.

[9] S. Chen, J. Entakli, M. Bonnard, E. Berton, and J. B. De Graaf, "Functional Corticospinal Projections from Human Supplementary Motor Area Revealed by Corticomuscular Coherence during Precise Grip Force Control," PLoS One, vol. 8, no. 3, 2013.

[10] F. Tadel, S. Baillet, J. C. Mosher, D. Pantazis, and R. M. Leahy, "Brainstorm: A user-friendly application for MEG/EEG analysis," Comput. Intell. Neurosci., vol. 2011, 2011.

[11] B. D. Van Veen, W. van Drongelen, M. Yuchtman, and A Suzuki, "Localization of brain electrical activity via linearly constrained minimum variance spatial filtering.," IEEE Trans. Biomed. Eng., vol. 44, no. 9, pp. 867-880, 1997.
[12] J. F. Hipp, D. J. Hawellek, M. Corbetta, M. Siegel, and A. K Engel, "Large-scale cortical correlation structure of spontaneous oscillatory activity," Nat. Neurosci., 2012.

[13] G. L. Colclough, M. J. Brookes, S. M. Smith, and M. W. Woolrich, "A symmetric multivariate leakage correction for MEG connectomes," Neuroimage, vol. 117, pp. 439-448, 2015.

[14] O. V. Lie and P. van Mierlo, "Seizure-Onset Mapping Based on Time-Variant Multivariate Functional Connectivity Analysis of High-Dimensional Intracranial EEG: A Kalman Filter Approach,” Brain Topogr., vol. 30, pp. 46-59, 2017.

[15] K. Kostoglou, C. T. Debert, M. J. Poulin, and G. D. Mitsis, "Nonstationary multivariate modeling of cerebral autoregulation during free-breathing and hypercapnic conditions," Med. Eng. Phys., vol. 36, pp. 592-600, 2014.

[16] K. Kostoglou and G. D. Mitsis, "Modelling of multiple-input, time-varying systems with recursively estimated basis expansions," (submitted).

[17] S. M. Kay, Modern spectral estimation. Pearson Education India, 1998.

[18] C. Porcaro, F. Zappasodi, P. M. Rossini, and F. Tecchio, "Choice of multivariate autoregressive model order affecting real network functional connectivity estimate," Clin. Neurophysiol., vol. 120, no. 2, pp. 436-448, 2009.

[19] K. Deep, K. P. Singh, M. L. Kansal, and C. Mohan, "A real coded genetic algorithm for solving integer and mixed integer optimization problems," Appl. Math. Comput., vol. 212, no. 2, pp. 505-518, 2009.

[20] J. M. Kilner, S. N. Baker, S. Salenius, R. Hari, and R. N. Lemon, "Human cortical muscle coherence is directly related to specific motor parameters.," J. Neurosci., vol. 20, no. 23, pp. 8838-8845, 2000

[21] J. Ushiyama, T. Suzuki, Y. Masakado, K. Hase, A. Kimura, M Liu, and J. Ushiba, "Between-subject variance in the magnitude of corticomuscular coherence during tonic isometric contraction of the tibialis anterior muscle in healthy young adults," $J$. Neurophysiol., vol. 106, no. 3, pp. 1379-1388, 2011.

[22] J.-M. Schoffelen, R. Oostenveld, and P. Fries, "Imaging the human motor system's beta-band synchronization during isometric contraction," Neuroimage, vol. 41, no. 2, pp. 437-447, 2008 